\title{
Gestión de simplificación administrativa en el desarrollo de las universidades públicas
}

\author{
Msc. Edman Junior Silva Huamantumba \\ jrsilva010889@gmail.com
}

\author{
Dr. José Manuel Delgado Bardales \\ manueldelgado1506@hotmail.com
}

\section{RESUMEN}

La investigación se realizó con el objetivo de conocer las características de la gestión de simplificación administrativa en el desarrollo de las Universidades públicas. La investigación es descriptiva, con enfoque cualitativo. La muestra estuvo conformada por artículos científicos; como técnica se usó el análisis bibliográfico y como instrumentos la guía de análisis de revisión bibliográfico, haciendo uso del método deductivo. Teniendo como resultados que el $70 \%$ hace referencia a la simplificación administrativa que está basada en procesos de tiempo en brindar el servicio, procesos administrativos y costos. El 30\%, menciona que la gestión de simplificación administrativa debe aplicar tecnología virtual que parte desde políticas internas institucionales con miras a mejorar los servicios con tramites accesibles y menos costosos, generando desarrollo a las instituciones gubernamentales. Concluyendo que la simplificación administrativa debe estar acompañada de tecnología, con programas que faciliten los procesos en menos tiempo, disminuyendo trámites, carga documental administrativa y los costos, para generar desarrollo en la institución. Además, la simplificación administrativa en las instituciones públicas es un requisito primordial para mejorar su competitividad; sin embargo, se necesitan mejorar los procesos de simplificación, con trámites menos burocráticos y servicios oportunos al ciudadano.

Palabras clave: Eficiencia, eficacia, gestión, procesos, simplificación administrativa. 


\title{
Administrative simplification management in the development of public universities
}

\begin{abstract}
The research was carried out with the objective of knowing the characteristics of the management of administrative simplification in the development of public universities. The research is descriptive, with a qualitative approach. The sample consisted of scientific articles; The bibliographic analysis was used as a technique and the bibliographic review analysis guide as instruments, making use of the deductive method. Having as results that $70 \%$ refer to administrative simplification that is based on time processes in providing the service, administrative processes and costs. 30\% mention that the management of administrative simplification must apply virtual technology that starts from internal institutional policies with a view to improving services with accessible and less expensive procedures, generating development for government institutions. Concluding that administrative simplification must be accompanied by technology, with programs that facilitate processes in less time, reducing procedures, administrative documentation burden and costs, to generate development in the institution. Furthermore, administrative simplification in public institutions is a primary requirement to improve their competitiveness; however, simplification processes need to be improved, with less bureaucratic procedures and timely services to citizens.
\end{abstract}

Keywords: Efficiency, effectiveness, management, processes, administrative simplification.

Artículo recibido: 03 nov. 2020 Aceptado para publicación: 07 dic. 2020 Correspondencia jrsilva010889@gmail.com Conflictos de Interés: Ninguna que declarar 


\section{Introducción}

La investigación, tiene como propósito conocer las características de la simplificación las características de la simplificación administrativa y su funcionamiento en las universidades públicas; con ello, proponer mejoras mediante las conclusiones para superar el problema. Las instituciones públicas presentan problemas en lo que concierne a trámites, tiempo y recursos económicos, por ello, se presenta el problema a nivel internacional y nacional.

La simplificación administrativa en las instituciones públicas de los países de América Latina, tiende a la eliminar largos trámites, con demoras y costos que se podrían simplificar en menos pasos, ahorrando en todo a la sociedad y que conllevará a un mejor manejo de las instituciones gubernamentales. La simplificación administrativa en las instituciones públicas es un requisito primordial para mejorar su competitividad, es por ello que la mayoría no han logrado avanzar debido a que los trámites son burocráticos y los servicios administrativos no son tan oportunos para los usuarios, a pesar de existir un marco normativo de calidad y mejoramiento continuo, es por ello que se deben revisar los lineamientos de políticas con miras a que garanticen el ajuste institucional que ayuden al cumplimiento de objetivos y calidad del servicio al usuario (Muñoz, 2011, p. 21).

En el Perú, las instituciones públicas como las universidades, también presentan deficiencias en los trámites documentarios para brindar los servicios al ciudadano, con largas colas para ser atendidos, acarreando costos al estado. Es decir, la simplificación administrativa debe mejorar los procesos y reducir costos, tiempos y trámites, relacionados a mejorar la eficiencia y eficacia de las instituciones, con menos procesos administrativos (Calagua, 2018, p. 11).

Por su parte, el gobierno regional de San Martín, con resolución N 523 - 2018- GRSM/GR, implantó el proceso de modernización en la gestión de turno, con el fin de mejorar en la calidad de servicios que brinda al ciudadano, optimizando los recursos y el beneficio social (GORESAM, 2018). Sin embargo, a pesar de ello, siguen existiendo problemas de demoras en los trámites administrativos, sin implementar correctamente las normas regionales. Se entiende que la simplificación implementada orienta y optimiza los servicios brindados a la población, respaldado con un buen marco normativo y herramientas adecuadas que mejoren la labor administrativa; sin embargo, falta mucho por mejorar.

Después de ello se tienen los siguientes antecedentes: 
Bueno. (2019). En su investigación "Acerca del fenómeno legal de la simplificación administrativa, ¿facilidad o restricción? (artículo científico), Universidad Carlos III de Madrid, España. El tipo de investigación fue descriptiva simple: la población y muestra estuvo conformada por documentos administrativos, como técnica el análisis documental e instrumento la guía de análisis. Concluye que la comunicación transversal es una herramienta importante en la simplificación administrativa. Para ello se tuvo la articulación de una triada de elementos como la parte normativa, orgánica y procedimental, que dio lugar a una investigación sobre las disposiciones normativas que regulan el fenómeno y permitirán el acceso a la información sin trabas ni demoras (P. 33).

Así mismo, Lozano (2015), en su investigación “El BOE como tablón edictal único y otras medidas de simplificación administrativa" (artículo científico), España. La investigación fue no experimental y descriptiva, de diseño no experimental; la muestra estuvo conformada por documentos bibliográficos; la técnica fue el análisis documental y el instrumento fue el análisis bibliográfico. Llegando a la conclusión que el $\mathrm{BOE}$ es un programa de reordenamiento de organismos públicos que mejora la simplificación administrativa. Ayuda a mejorar la eficiencia, educir tiempo en los procesos de controles y registros administrativos, hacerlos más sencillos y accesibles (P. 117)

A su vez, Chain, C. (2017), en su investigación “Gestión institucional y simplificación administrativa en la Universidad Nacional de San Antonio Abad del Cusco, 2017”. (artículo científico), Universidad César Vallejo, Perú. El tipo de investigación fue no experimental, con diseño descriptivo simple

La investigación fue descriptivo correlacional, con enfoque mixto, y de diseño no experimental; la población y muestra fueron los administrativos de la universidad; la técnica fue la entrevista y el instrumento el cuestionario. Concluye que, El factor Gestión institucional influye en la Simplificación administrativa, debido a que esta última se implementa sobre los principios de gestión pública, los que a su vez influyen de manera directa y dependiente sobre los procesos de simplificación y modernización de la gestión estatal, donde la gestión del talento humano y la incorporación de las nuevas tecnologías como el Internet en los procedimientos administrativos.

\section{Teorías relacionadas al tema}

\section{Simplificación administrativa}


Las instituciones del estado, cada vez han ido buscando la manera de implementar herramientas que permitan llegar al ciudadano de manera exitosa. Donde los procesos han ido a acompañadas a disminuir cargas administrativas y procedimentales para poder acceder a los mercados desde los años 90 (López, 2013, p. 126).

Para ello, la gestión hace referencia a las actividades que desarrollan los directivos de una organización o empresa para conseguir una adecuada productividad con eficacia y eficiencia. Sus resultados dependen de sus habilidades o la aplicación de métodos o técnicas con sus colaboradores mediante procesos establecidos (Trayter, 2016, p. 32).

La simplificación administrativa facilita los procedimientos en las institucione gubernamentales para asegurar la eficacia y eficiencia. Engloba procesos para la reducción de pasos y facilitar la administración en la parte normativa, orgánica y procedimental, relacionados entre sí, con el objetivo de usar racionalmente los recursos escasos y ser más eficientes en tiempos, por ello la simplificación administrativa es una excelente herramienta de competitividad de la institución (Torres, 2018. P. 26).

La simplificación administrativa, articula procesos de planificación, organización, dirección y control, aplicados en los procesos para reducir costos, y tiempo en trámites, mejorando la eficiencia en atención, costos de procedimientos y procesos administrativos en general. También está basada en políticas internas de las instituciones para mejorar los servicios brindados con eficiencia social (Calagua, 2018, p. 23).

La importancia de la simplificación administrativa radica en la facilidad de los procedimientos administrativos, haciéndolos menos complicados, más eficientes en menos tiempo. La simplificación administrativa es más dinámica y operativa en los ámbitos de simplificación normativa, orgánica y procedimental relacionados entre sí (Esparza, 2013, p. 21).

Después de lo mencionado en la realidad problemática, se formula el siguiente problema de investigación:

¿Cómo es la gestión de simplificación administrativa en el desarrollo de las Universidades públicas?

Como objetivo se plantea lo siguiente:

Conocer las características de la gestión de simplificación administrativa en el desarrollo de universidades públicas. 
Como propósito investigativo es, conocer las características de la simplificación administrativa y su funcionamiento para el desarrollo en las universidades públicas; con ello, proponer mejoras mediante las conclusiones para superar el problema.

El estudio se justifica ya que las conclusiones ayudarán a mejorar la implementación de políticas que tienen que ver con la gestión de simplificación administrativa y mejorar los procesos del mismo en favor de la comunidad estudiantil de las universidades públicas. A su vez, ayudarán a los estudiantes a tener mejor rapidez en la atención y trámites administrativos, así como disminución de costos y tiempo a los estudiantes y facilitando el trabajo de los colaboradores y demás usuarios de las universidades públicas. También aportará teóricamente y metodológicamente mediante la técnica e instrumentos propias de investigación descriptiva que serán de referencia para otros estudios similares.

\section{Material y métodos}

La investigación es no experimental, de tipo aplicada, ya que se utilizarán teorías existentes y estudios respectivos para comprobar la hipótesis. En lo que se refiere al diseño de investigación es descriptiva simple, ya que se describirá la variable de simplificación administrativa.

El enfoque de investigación fue cualitativo, ya que no se recurrió a la cuantificación numérica para comprobar la hipótesis; así mismo, el análisis final termina con la descripción para interpretar el problema de estudio.

El método utilizado fue el hipotético deductivo, partiendo de hechos generales para llegar a hechos generales mediante las conclusiones del estudio referido a la simplificación administrativa. Los materiales utilizados fueron libros, laptop, referencias en línea y tablas de análisis para la investigación.

La muestra estuvo conformada por artículos científicos a nivel internacional y nacional, con contenido original y confiable respecto a la variable de estudio, la misma que fue fundamental para analizar los respectivos resultados y conclusiones. Los datos de dichas investigaciones son confiables, que responden al objetivo de estudio, sin alterar los resultados de estudio como parte de la ética de resultados originales, incluyendo las citas de los autores.

La técnica utilizada fue el análisis bibliográfico de artículos científicos respecto a la variable de estudio. Como instrumentos se usó la guía de análisis bibliográfico mediante tablas respectivas por cada autor, donde contiene los principales resultados y conclusiones, permitiendo realizar el análisis crítico, llegando a conclusiones generales de los diferentes artículos científicos. 
El proceso de análisis de investigación fue mediante tablas de análisis de cada investigación utilizada, donde se procedió al análisis de los principales resultados y conclusiones, llenado a una conclusión final de acuerdo al objetivo de estudio.

Referente a la ética de investigación, se respetó la autoría de los autores empleados en la investigación, citando a cada uno de ellos mediante el APA.

\section{Resultados}

Los resultados de la investigación están en función a los cuadros de revisión de diferentes autores, mostrados en tablas a continuación: 
Tabla 01:

Datos de publicación de autor 01

\begin{tabular}{|c|c|c|c|c|c|}
\hline 1. Autor & Año & \multicolumn{2}{|c|}{ Título } & Ubicación & Volumen y numero de revista \\
\hline Fernández, R. & 2019 & $\begin{array}{l}\text { Hacia la simpli } \\
\text { procedimental: } \\
\text { procedimiento a }\end{array}$ & $\begin{array}{l}\text { ificación y la } \\
\text { Las nue } \\
\text { abierto. }\end{array}$ & $\begin{array}{l}\text { transparencia en materia } \\
\text { as modalidades del }\end{array}$ & $\begin{array}{l}\text { Revista Gabilex N } N^{\circ} \text { Extraordinario } \\
\text { Marzo 2019. Vol I. }\end{array}$ \\
\hline \multicolumn{6}{|c|}{ DESARROLLO } \\
\hline $\begin{array}{l}\text { Metodología } \\
\text { utilizada }\end{array}$ & $\begin{array}{l}\text { Población y } \\
\text { Muestra }\end{array}$ & $\begin{array}{l}\text { Técnicas e } \\
\text { instrumento }\end{array}$ & Parte é & Resultados & Conclusiones \\
\hline Descriptiva & $\begin{array}{c}\text { Revisión } \\
\text { documentari } \\
\text { a }\end{array}$ & $\begin{array}{c}\text { Ficha de } \\
\text { recolección } \\
\text { de datos de } \\
\text { Publicacion } \\
\text { es }\end{array}$ & $\begin{array}{c}\text { Código de } \\
\text { ética en } \\
\text { investigació } \\
\mathrm{n}\end{array}$ & $\begin{array}{l}\text { La simplificación administrativa no es ágil en la } \\
\text { institución. Con todo, en la medida en que se } \\
\text { cumpla la obligación de llevar a cabo una } \\
\text { contratación verdadera y completamente } \\
\text { electrónica, donde mediante el diseño en la fase } \\
\text { de adjudicación permite una mayor simplificación } \\
\text { y agilidad de tramitación. }\end{array}$ & $\begin{array}{l}\text { stión de simplificación mediante el } \\
\text { agiliza y simplifica los } \\
\text { limientos administrativos, mediante } \\
\text { ntratación electrónica. Tiene los } \\
\text { os de lograr reducir los tiempos, } \\
\text { rocesos cortos y eficiencia en las } \\
\text { taciones con el estado. }\end{array}$ \\
\hline
\end{tabular}

Fuente: Elaboración propia. 


\section{Tabla 02}

Datos de publicación de autor 02.

\section{Autor} Año
Nombre de la Investigación
Revista donde se ubica la Publicación

\section{Volumen Y Numero}

Cochrane Database of Systematic Reviews 2010, Issue 4. Art. No.: CD008495.
Simplificación administrativa y modificación o supresión de las

Trayter, J. https://dialnet.uniri oja.es/servlet/articu lo?codigo $=572875$ 9 DOI: $10.1002 / 14651858 . C D 008495$.

técnicas de limitación o

Cochrane Database of Systematic Revi

intervención.

España

4. Art. No.: CD008495.

DOI: $10.1002 / 14651858 . C D 008495$.

Revista Aragonesa de

Pública, ISSN 1133-4797, № 47-48, págs. 8-44

\begin{tabular}{|c|c|c|c|c|c|}
\hline \multicolumn{6}{|c|}{ DESASRROLLO } \\
\hline $\begin{array}{l}\text { Metodología de la } \\
\text { investigación }\end{array}$ & $\begin{array}{l}\text { Población } \\
\text { y Muestra }\end{array}$ & $\begin{array}{c}\text { Técnicas e } \\
\text { instrumentos }\end{array}$ & Parte ética & a $\quad$ Resultados & Conclusiones \\
\hline Cualitativo & $\begin{array}{l}\text { Revisión } \\
\text { documenta } \\
\text { ria }\end{array}$ & $\begin{array}{l}\text { Ficha de } \\
\text { recolección } \\
\text { de datos de } \\
\text { Publicacione } \\
\quad \text { s }\end{array}$ & $\begin{array}{cc} & \mathrm{I} \\
\text { Código de } & \mathrm{t} \\
\text { ética en } & 1 \\
\text { investigación } & \mathrm{f} \\
& \mathrm{c} \\
& \mathrm{i}\end{array}$ & $\begin{array}{l}\text { La simplificación administrativa, está } \\
\text { basada en las normas administrativas de } \\
\text { las instituciones. El punto de partida es } \\
\text { facilitar los trámites en lo que se refiere } \\
\text { a licencias o autorizaciones de interés } \\
\text { institucional. }\end{array}$ & $\begin{array}{l}\text { La simplificación administrativa está } \\
\text { basada en normas institucionales y } \\
\text { principios de comunicación para } \\
\text { cumplir con los procesos en menos } \\
\text { tiempo con mayor efectividad. }\end{array}$ \\
\hline
\end{tabular}

Fuente: Elaboración propia

Tabla 03

Datos de publicación de autor 03.

\begin{tabular}{cclcc}
\hline 3. Autor & Año & Título & Ubicación & Volumen y número de la revista \\
\hline \multirow{2}{*}{ Vega, Y. } & & Simplificación de requisitos para disminuir el & http://repositorio.upn.edu.pe/han & Repositorio Institucional, \\
& 2018 & tiempo de obtención de licencia de funcionamiento & dle/11537/13027?show=full & Universidad Privada del \\
& & para actividades económicas en la Municipalidad de & Carmen de la Legua Reynoso, Calla, 2016. & Perú
\end{tabular}




\section{CONTENIDO DE LA PUBLICACIÓN}

\begin{tabular}{|c|c|c|c|c|}
\hline $\begin{array}{l}\text { Metodología de la } \\
\text { investigación }\end{array}$ & $\begin{array}{l}\text { Población } \\
\text { y Muestra }\end{array}$ & $\begin{array}{c}\text { Técnica e } \\
\text { instrumento }\end{array}$ & Parte ética & Conclusiones \\
\hline Descriptivo. & $\begin{array}{l}\text { Documento } \\
\text { s sobre el } \\
\text { tema. }\end{array}$ & $\begin{array}{l}\text { Ficha de } \\
\text { recolección de } \\
\text { datos de } \\
\text { Publicaciones }\end{array}$ & $\begin{array}{l}\text { Código de ética } \\
\text { en investigación }\end{array}$ & $\begin{array}{l}\text { La Municipalidad de Carmen, con la La gestión de simplificación } \\
\text { finalidad de asegurar la administrativa reduce tiempo de servicio, } \\
\text { simplificación administrativa de este menos procesos administrativos y menos } \\
\text { trámite, modifico las funciones de la costos, generando satisfacción en los } \\
\text { Subgerencia de Desarrollo para usuarios. Con ello las instituciones } \\
\text { mejorar los servicios de información cumplen sus metas y son más } \\
\text { y trámites rápidos a través de su competitivos. } \\
\text { página web. }\end{array}$ \\
\hline \multicolumn{5}{|c|}{$\begin{array}{l}\text { Fuente: Elaboración propia } \\
\text { Tabla } \mathbf{0 4} \\
\text { Datos de publicación de autor } 04\end{array}$} \\
\hline 4. Autor & Año & \multirow{2}{*}{\multicolumn{2}{|c|}{$\begin{array}{c}\text { Nombre de la Investigación } \\
\text { El BOE como tablón edictal único } \\
\text { otras medidas de simplificación } \\
\text { administrativa }\end{array}$}} & volumen y número de la investigación \\
\hline Lozano, B. & 2014 & & & $\begin{array}{l}\text { yttps://dialnet.unirioja.es/servlet/articulo? } \\
\begin{array}{l}\text { codigo=4824537 Diario La L Ley, ISSN 1989- } \\
\text { España }\end{array} \\
6913, N^{\circ} 8398,2014 .\end{array}$ \\
\hline \multicolumn{5}{|c|}{ DESARROLLO } \\
\hline Metodología & $\begin{array}{l}\text { Población y } \\
\text { Muestra }\end{array}$ & $\begin{array}{c}\text { Técnicas e } \\
\text { instrumentos }\end{array}$ & Parte ética & Conclusiones \\
\hline Investigación descriptiva & $\begin{array}{l}\text { Documento } \\
\text { s } \\
\text { administrati } \\
\operatorname{vos}\end{array}$ & $\begin{array}{c}\text { Análisis } \\
\text { documental }\end{array}$ & $\begin{array}{cl} & \mathrm{C} \\
\text { Consentimiento } & \text { re } \\
\text { informado. } & \text { ac } \\
& \text { ge } \\
& \text { pc } \\
\text { re }\end{array}$ & $\begin{array}{l}\text { Como resultados tuvo que, la ley } \\
\text { iene cuatro elementos nuevos } \\
\text { ienejorar la eficiencia. Reducir tiempo en los } \\
\text { eferidos a la simplificación procesos de controles y registros } \\
\text { administrativa y transparentando la administrativos, hacerlos más sencillos y } \\
\text { gestión. A su vez, con el BOE, se accesibles. } \\
\text { oudo reordenar algunas reformas y } \\
\text { regímenes. }\end{array}$ \\
\hline
\end{tabular}

Fuente: Elaboración propia 
Tabla 05

Datos de publicación de autor 05

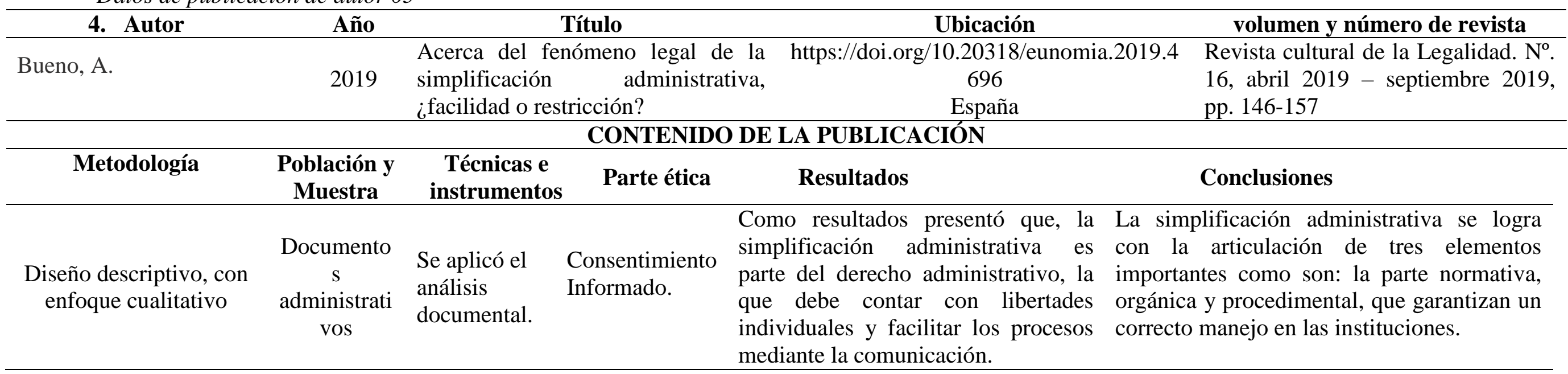

Fuente: Elaboración propia

Tabla 06

Datos de publicación de tabla 06
4. Autor
Año Nombre de la Investigación Gestión institucional y
Chain, C. simplificación administrativa en
2017 la Universidad nacional de San Antonio Abad, Cusco, 2017.
Volumen y número de revista
http://repositorio.ucv.edu.pe/handle/UC
V/19910
Repositorio Universidad César Vallejo Perú

\begin{tabular}{|c|c|c|c|c|c|}
\hline \multicolumn{6}{|c|}{ DESARROLLO } \\
\hline Metodología & $\begin{array}{l}\text { Población y } \\
\text { Muestra }\end{array}$ & $\begin{array}{c}\text { Técnicas e } \\
\text { instrumentos }\end{array}$ & Parte ética & Resultados & Conclusiones \\
\hline $\begin{array}{l}\text { Descriptivo } \\
\text { correlacional }\end{array}$ & $\begin{array}{l}\text { Administrati } \\
\text { vos de la } \\
\text { Universidad }\end{array}$ & Cuestionario & $\begin{array}{l}\text { Código de } \\
\text { ética en } \\
\text { investigac } \\
\text { ión. }\end{array}$ & $\begin{array}{l}\text { En los resultados } \\
\text { muestran un alto nivel } \\
\text { de insatisfacción por } \\
\text { parte del personal } \\
\text { administrativo, con }\end{array}$ & $\begin{array}{l}\text { La simplificación administrativa, tiene un elemento } \\
\text { importante que es la gestión institucional que ayudan a } \\
\text { mejorar el desarrollo de las instituciones y a la } \\
\text { modernización del estado. En esta intervienen } \\
\text { elementos como el talento humano, la tecnología como }\end{array}$ \\
\hline
\end{tabular}


procesos en un mayor el internet que facilita el trabajo.

tiempo y altos costos,

ocasionando malestar

en los usuarios.

Fuente: Elaboración propia

Tabla 07

Datos de publicación de autor 07

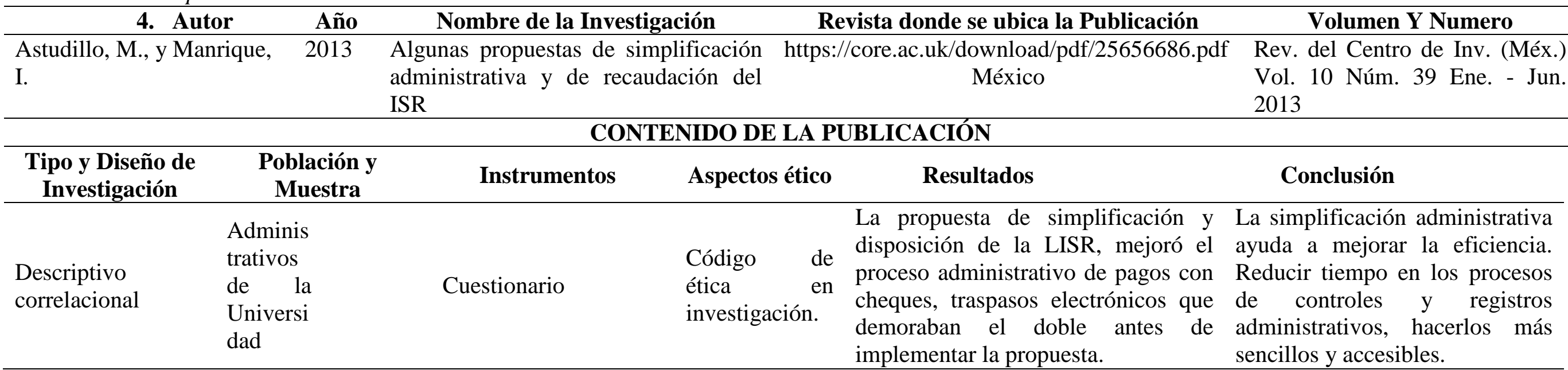

Fuente: Elaboración propia

Tabla 08

Datos de publicación 08

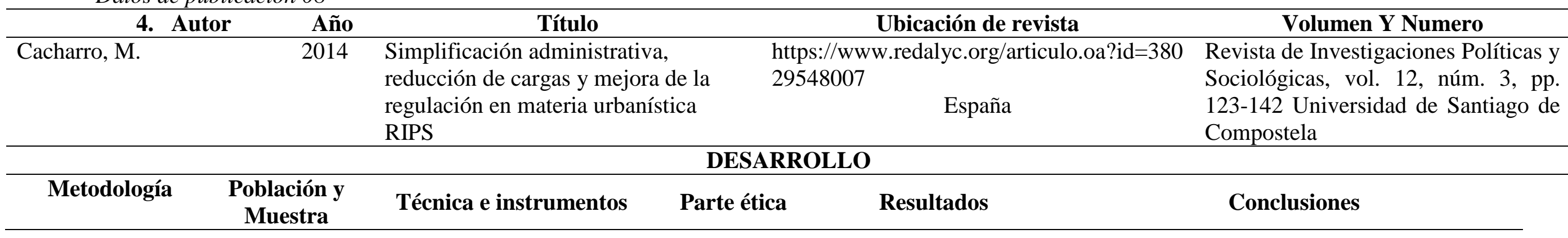




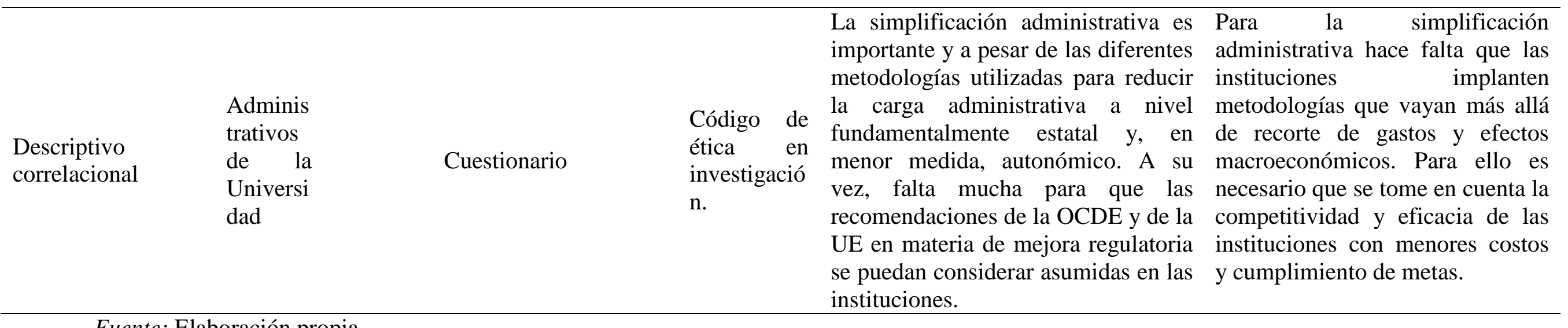

Fuente: Elaboración propia 


\section{Análisis de resultados}

Después de colocar los principales resultados en las tablas de análisis de los diferentes autores, pasamos al análisis respectivo de cada uno.

Fernández (2019), indica que la revisión documentaria arrojó como resultados que en la medida en que se cumpla la obligación de llevar a cabo una contratación verdadera y completamente electrónica, donde mediante el diseño en la fase de adjudicación permitirá una mayor simplificación y agilidad de tramitación (p. 12). Así mismo, la simplificación administrativa, está basada en las normas administrativas de las instituciones. El punto de partida es facilitar los trámites en lo que se refiere a licencias o autorizaciones de interés institucional (Trayter, 2016, p. 8),

Vega (2018), en sus resultados muestra que la simplificación disminuye el tiempo en un 30\% y costos en las actividades económicos de la Municipalidad (p. 13). Así mismo, Lozano (2014), en su investigación tiene resultados, la simplificación administrativa mejora el tiempo en el que se brinda el servicio, con menos procesos en la parte administrativa, menores costos que hacen mas competitivas a las instituciones; engloba la parte normativa para la transparencia de la gestión (p. 9).

Por su parte, Bueno (2019), tiene como resultados que la simplificación administrativa articula tres elementos como lo normativo, orgánico y procedimental que ayuda a desarrollar a las instituciones con una mejor comunicación (p. 12). Chain (2017), indica que los colaboradores administrativos están insatisfechos debido a la ineficiente simplificación administrativa, teniendo efectos negativos en los usuarios que hacen uso de los servicios y obstruyendo el trabajo institucional de los mismo (p. 14).

Astudillo y Manrique (2013), en su investigación menciona que la propuesta de simplificación y disposición de la LISR ayudó al proceso administrativo de pagos con cheques, traspasos electrónicos que demoraban el doble antes de implementar la propuesta. Con la propuesta de simplificación administrativa y mayor control de las operaciones hechas por los contribuyentes se pudo deducir los gastos e inversiones realizados (p. 9). Cacharro (2013), en su investigación, tiene como resultados que la simplificación administrativa es importante y a pesar de las diferentes metodologías utilizadas para reducir la carga administrativa a nivel fundamentalmente estatal y, en menor medida, autonómico (p. 11).

De todas las investigaciones se tuvo que el $70 \%$ hace referencia a la simplificación administrativa que está basada en procesos de tiempo en brindar el servicio, procesos administrativos y costos. Todos estos procesos en corto tiempo, hacen que las instituciones 
sean más competitivas, cumpliendo metas y mejorando su imagen ante los usuarios. Por otro lado, el 30\%, menciona que la gestión de simplificación administrativa debe aplicar tecnología virtual que parte desde políticas internas institucionales con miras a mejorar los servicios con tramites accesibles y menos costosos, generando desarrollo a las instituciones gubernamentales, corroborando.

\section{Discusión}

Después de realizar el análisis correspondiente de las investigaciones de artículos científicos, del total, el 90\% son de España y Perú, el 10\% son de México. Además, las investigaciones han aplicado el análisis bibliográfico y son de carácter descriptivo, teniendo los siguientes resultados.

Los resultados permitieron conocer las características de la gestión administrativa de las universidades públicas, en las que el 70\% indican que existe una alta influencia de la gestión de simplificación administrativa en la eficiencia en que las instituciones brindan mejores servicios, en menos tiempo, menos costos debido a los procesos cortos, haciendo mas competitivas a las instituciones. El 30\% de autores, indican que la gestión de simplificación administrativa está ligado a la aplicación de nuevas tecnologías, para ello debe existir una política sólida al respecto en las instituciones, con el propósito de disminuir los trámites, carga documental administrativa y los costos, con miras a generar desarrollo en la institución. En ese sentido, en concordancia con el 30\% de resultados, Fernández (2019), también hace énfasis a la tecnología mediante el PASA de contratación electrónica que simplifica los procedimientos administrativos. Contrariamente Trayter (2016), afirma que la simplificación administrativa está basada en normas institucionales y principios de comunicación para cumplir con los procesos en menos tiempo con mayor efectividad.

De la misma forma, Vega (2018), concuerda que la gestión de simplificación administrativa reduce tiempo de servicio, menos procesos administrativos y menos costos, generando satisfacción en los usuarios. Con ello las instituciones cumplen sus metas y son más competitivos. Así mismo, Lozano (2014), coincide que la simplificación administrativa ayuda a mejorar la eficiencia, reduciendo tiempo en los procesos de controles y registros administrativos, hacerlos más sencillos y accesibles.

Incluso, Astudillo y Manrique (2013), concuerda que la simplificación administrativa ayuda a mejorar la eficiencia. Reducir tiempo en los procesos de controles y registros administrativos, hacerlos más sencillos y accesibles. Si embargo, Bueno (2019), concuerda con el 30\% de 
resultados que la simplificación administrativa, es necesario tener en cuenta la triada de la normativa, elementos orgánicos y la parte de procedimientos, con el respaldo de la parte normativa que hace más eficientes a las instituciones.

Por último, Chain (2017), concuerda que la simplificación administrativa tiene un elemento importante que es la gestión institucional, para mejorar procesos de modernización del estado. Por su parte, Cacharro (2013), también concuerda en que las instituciones deben implantar metodologías que tome en cuenta la competitividad y eficacia de las instituciones con menores costos y cumplimiento de metas.

Así mismo, la simplificación administrativa falta ser abordada desde espacios de simplificación mediante sistemas desde las redes sociales y nuevas tecnologías, ayudando a disminuir procesos, en menos tiempo, cumpliendo con los objetivos de las instituciones, mediante procesos eficientes y menores costos, en beneficio de los usuarios, referido a procesos administrativos. Actualmente, falta mejorar los procesos mencionados, observando trámites burocráticos y los servicios administrativos no son tan oportunos para los usuarios.

\section{Conclusiones:}

Las investigaciones analizadas, ayudaron a conocer las características de la gestión de simplificación administrativa en el desarrollo de instituciones públicas de la siguiente manera: Concluye que la gestión de simplificación administrativa tiene un proceso de planificación desde políticas internas con miras a mejorar la calidad del servicio, para ello, está basada en procesos de tiempos de servicio, procesos administrativos y costos, haciendo que las instituciones cumplan sus metas y sean más competitivas brindando mayores satisfacciones a los usuarios.

A su vez, concluye que la simplificación administrativa debe estar acompañada de tecnología, con programas que faciliten los procesos en menos tiempo, disminuyendo trámites, carga documental administrativa y los costos, con miras a generar desarrollo en la institución. Además, la simplificación administrativa en las instituciones públicas es un requisito primordial para mejorar su competitividad; sin embargo, se necesitan mejorar los procesos de simplificación, con trámites menos burocráticos y servicios oportunos al ciudadano.

Por ello se sugiere una mejora en las políticas de las instituciones públicas como las Universidades para mejorar la simplificación administrativa en lo que se refiere a reducción de trámites, costos y procesos burocráticos, facilitando un mejor servicio al usuario y mejorando la competitividad de las instituciones. 


\section{Referencias}

Astudillo, M., y Manrique, E. (2013). Algunas propuestas de simplificación administrativa y de recaudación del ISR. Rev. del Centro de Inv. (Méx.) Vol. 10 Núm. 39 Ene. Jun. 2013, México. Recuperado de: http://eds.b.ebscohost.com/eds/pdfviewer/pdfviewer?vid=0\&sid=03fdd9 58-a7bf4e70-8827-cbbaf801b318\%40pdc-v-sessmgr05

Bueno, A. (2019). Acerca del fenómeno legal de la simplificación administrativa, ¿facilidad o restricción?. Eunomía. Revista en Cultura de la Legalidad. №. 16, abril 2019 septiembre 2019, pp. 146-157, España. Recuperado de: https://doi.org/10.20318/eunomia.2019.4696

Cacharro, M. (2013). Simplificación administrativa, reducción de cargas y mejora de la regulación en materia urbanística RIPS. Revista de investigaciones políticas y sociológicas, vol. 12, núm. 3, 2013, pp. 123-142 Universidad de Santiago de Compostela Santiago de Compostela, España. Recuperado de: https://www.redalyc.org/articulo.oa?id=38029548007

Calagua, M. (2018). La simplificación administrativa y la calidad de servicio en la superintendencia de transporte terrestre de personas, carga y mercancías - Jesús María 2017. (Tesis de post grado), Universidad César Vallejo, Lima. Recuperado de:

http://repositorio.ucv.edu.pe/bitstream/handle/UCV/17017/Calagua_MMF.pdf?se quence $=1 \&$ isAllowed $=\mathrm{y}$

Chain, C. (2017). Gestión institucional y simplificación administrativa en la Universidad Nacional de San Antonio Abad del Cusco, 2017. Repositorio UCV. Recuperado de: http://repositorio.ucv.edu.pe/handle

Fernández, R. (2019). Hacia la simplificación y la transparencia en materia procedimental: Las nuevas modalidades del procedimiento abierto. Junta de Comunidades de Castilla La Mancha. Revista Gabilex $N^{o}$ Extraordinario, España. Recuperado de: http://gabilex.castillalamancha.es

Gobierno Regional San Martín - GORESAM (2018). Resolución ejecutiva regional. Gerencia general. Recuperado de: https://www.regionsanmartin.gob.pe/OriArc.pdf?id=110264 
López, C. (2013). Simplificación administrativa, reducción de cargas y mejora de la regulación en materia urbanística. RIPS. Revista de Investigaciones Políticas y Sociológicas, vol. 12, núm. 3, pp. 123-142. Universidad de Santiago de Compostela Santiago de Compostela, España. https://www.redalyc.org/articulo.oa?id=38029548007

Lozano, B. (2015). El BOE como tablón edictal único y otras medidas de simplificación administrativa. Diario La Ley, $\mathrm{N}^{\circ}$ 8398. España. Recuperado de: https://dialnet.unirioja.es/servlet/articulo? codigo $=4824537$

Muñoz, W. (2011). PERÚ: La simplificación administrativa en el marco del proceso de modernización del estado. Universidad Católica Sedes Sapientiae, Perú. Recuperado de: http://cidir.ucss.edu.pe/images/investigaciones/perusimplificacion-administrativa-modernizacion-estado.pdf

Torres, R. (2018). La gestión administrativa y el control interno en el área administrativa del SENASA 2017. (Tesis de post grado, Universidad César Vallejo). Perú. http://repositorio.ucv.edu.pe/handle/UCV/21086

Trayter, J. (2016). Simplificación administrativa y modificación o supresión de las técnicas de limitación o intervención. Revista Aragonesa de Administración Pública, ISSN 1133-4797, No 47-48, págs. 8-44. Recuperado de: https://dialnet.unirioja.es/servlet/articulo?codigo=5728759, España.

Vega, Y. (2018). Simplificación de requisitos para disminuir el tiempo de obtención de la licencia de funcionamiento para actividades económicas en la municipalidad de Carmen de la Legua Reynosos, Callao año 2016. (Tesis de pre grado), Universidad Privada del norte, Perú. Recuperado de: http://repositorio.upn.edu.pe/handle/11537/13027?show=full 